\title{
Multi-Hop Connectivity Probability in Infrastructure-Based Vehicular Networks
}

\author{
Wuxiong Zhang, Yu Chen, Yang Yang, Senior Member, IEEE, Xiangyang Wang, Yu Zhang, Xuemin Hong, \\ Guoqiang Mao, Senior Member, IEEE
}

\begin{abstract}
Infrastructure-based vehicular networks (consisting of a group of Base Stations (BSs) along the road) will be widely deployed to support Wireless Access in Vehicular Environment (WAVE) and a series of safety and non-safety related applications and services for vehicles on the road. As an important measure of user satisfaction level, uplink connectivity probability is defined as the probability that messages from vehicles can be received by the infrastructure (i.e., BSs) through multi-hop paths. While on the system side, downlink connectivity probability is defined as the probability that messages can be broadcasted from BSs to all vehicles through multi-hop paths, which indicates service coverage performance of a vehicular network. This paper proposes an analytical model to predict both uplink and downlink connectivity probabilities. Our analytical results, validated by simulations and experiments, reveal the trade-off between these two key performance metrics and the important system parameters, such as BS and vehicle densities, radio coverage (or transmission power), and maximum number of hops. This insightful knowledge enables vehicular network engineers and operators to effectively achieve high user satisfaction and good service coverage, with necessary deployment of BSs along the road according to traffic density, user requirements and service types.
\end{abstract}

Index Terms - Vehicular ad-hoc Network (VANET), Wireless Access in Vehicular Environment (WAVE), IEEE 802.11p, IEEE 1609, Connectivity Probability

\section{INTRODUCTION}

$\mathbf{V}$ EHICLES started to emerge as an important way of traveling in Chinese daily life during the past decade. Since 2005, the number of private car ownership keeps increasing by around $30 \%$ every year and has reached 34.43 million by the end of 2010 [1]. Statistics also show that the average commuting time in major cities of China is more than 60 minutes [2]. As people have to spend more and

Manuscript received 15 May 2011; revised 28 December 2011.

W. Zhang and Y. Yang are with Shanghai Research Center for Wireless Communications, Shanghai Institute of Microsystem and Information Technology (SIMIT), Chinese Academic of Sciences (CAS), Key Laboratory of Wireless Sensor Network \& Communication, SIMIT, CAS and Graduate University of Chinese Academic of Sciences, China (e-mail: \{wuxiong.zhang, yang.yang\}@shrcwc.org).

Y. Chen is with University College London (UCL), U.K. (e-mail: uceec00@ee.ucl.ac.uk).

X. Wang is with Southeast University, China (e-mail: xywang@seu.edu.cn).

Y. Zhang is with the National Engineering Research Center for Broadband Networks and Applications in Shanghai, China (e-mail: yuzhang@hotmail.co.uk).

$\mathrm{X}$. Hong is with Xiamen University, Xiamen, China (e-mail: xuemin.hong@gmail.com).

G. Mao is with School of Electrical and Information Engineering, University of Sydney and NICTA, Australia (e-mail: guoqiang.mao@sydney.edu.au).

Digital Object Identifier 10.1109/JSAC.2012.1205xx. more time on road, there is a strong demand to provide a media rich broadband wireless communications system to passengers. China has been carrying out an ambitious plan to build a national TD-LTE mobile network. Meanwhile, the IEEE $802.11 \mathrm{p}$ and 1609 standards are under development to support Wireless Access in Vehicular Environment (WAVE) in VANETs and to deliver safety and non-safety applications to vehicles on the road [3][4], and wireless system for high speed trains are also under research [5]. However, how to provide easy and effective communications between vehicles with dynamic mobility and as well as between vehicles and roadside base stations still remains an open and challenging area.

Various challenges need to be solved before an intelligent vehicular network comes into reality. Due to the random location and mobility of vehicles, and the limited roadside resources, the connectivity among vehicles and BSs are not naturally guaranteed. From passengers' perspective, the uplink connectivity probability, defined as the probability that messages from vehicles can be received by BSs through multihop paths, is a critical performance metric and is arguably the most important service requirement that directly affects users' experiences and satisfaction levels in data transmissions. From roadside infrastructures' perspective, the downlink connectivity probability, defined as the probability that messages can be broadcasted from BSs to all vehicles through multihop paths is important and is related to the service coverage of a vehicular network that determines the efficiency/quality broadcasting services (from a BS to many vehicles), such as safety and emergency information dissemination from a traffic manager or a network operator to all vehicles. Several ways can be used to improve both the uplink and downlink connectivity probabilities including increasing the radio coverage (or transmission power) of BSs, deploying more BSs or allowing multi-hop communications. However, the inter-cell interference may increase when the transmission power of BSs increases or the number of BSs increases. The more dense deployment of base stations will cost operators more at up-front investments and maintenance, hence multihop communications becomes an good solution for increasing the chance of accessibility when a vehicle cannot directly communicate with any BS (e.g., it is located in a coverage gap between adjacent BSs) by enabling it to rely on its neighboring vehicles to forward/relay data packets to/from a nearby BS. Although using multi-hop communications can greatly improve the connectivity probability, it will increase 
transmission delay, the complexity and system overhead of the routing algorithm and so on. Therefore, the maximum number of hops a path can go through should be up bounded. Finally, the probabilities are affected by real wireless channels (i.e., fadings) as well.

The connectivity probability in VANETs has been widely studied. Factors such as traffic flow models (e.g., individual mobility models [22], random waypoint model [20], Poisson distribution model [8], uniform distribution model), communication models (e.g., protocol model and physical model [19]), dimensions of network (e.g., one-dimensional, 2-dimensional network), and whether infrastructure is needed are considered most frequently. List just a few very closely related work: for ad-hoc-like networks, the authors in [7] derived a formula for one-dimensional MANET with vehicles uniformly distributed under a simple communication model (protocol model). The authors in [9] addressed connectivity problem in ad-hoc network under a realistic wireless channel model with shadowing and fading effects (physical communications model). In addition, closed-form formula for connectivity in linear ad-hoc network was calculated in [10] under a novel mobility model using multi-dimensional hypercube and hyperplane method. For infrastructure-based vehicle networks, connectivity probability formula for a vehicle-to-BS connection (defined as uplink connectivity probability in this paper) was derived in [11] and [21] under Poisson distribution and protocol model.

Those work above are insightful and important, however results for ad-hoc-like networks cannot be directly used in infrastructure-based networks, and results for infrastructurebased networks only focus on simple communication models (i.e., protocol models). Our work considers a realistic scenario wherein wireless devices at roadside BSs and moving vehicles have different coverage and capacity, and for the first time, we differentiate and derive uplink and downlink connectivity probabilities for infrastructure-based vehicular networks. Moreover, we use a Poisson traffic model and a log-normal shadow fading model for performance analysis in this paper, but our analytical approach can be easily extended to study connectivity probability under other traffic and channel models. Our work can be regarded as an integration, enhancement and extension of those work. Finally, we have already done some work to derive 2-hop connectivity probability for infrastructure-based VANETs [12] and [13], and it is straightforward to extend our work above to derive multi-hop connectivity probability for infrastructure-based VANETs. A recent closely related work in [6] provided an analysis framework for probabilistic delay for the delivery of vehicle-to-infrastructure packet under the concept of disrupted connectivity for highways with low density. Our work is complementary to this work, and focuses on the uplink and downlink connectivity performance in multi-hop scenarios. In this paper, we firstly derive and analyze the connectivity probability between a pair of arbitrary base station and vehicle (which can be regarded as connectivity probability in ad-hoclike networks) and then we move forward to study the uplink and downlink connectivity probabilities for infrastructurebased VANETs. The trade-off between some key performance metrics and some important system parameters, such as BS and vehicle densities, radio coverage (or transmission power), and maximum number of hops, are fully investigated. This insight knowledge enables vehicular network engineers and operators to effectively achieve high user satisfaction and good service coverage, with only necessary deployment of BSs along the road according to traffic density, user requirements and service types. Finally, our analytical results are validated by both computer-based simulations and experiments (realtraffic-flow-based simulations).

The rest of this paper is organized as follows. System model of infrastructure-based vehicular networks is defined in section II. The connectivity probability between an arbitrary pair of BS and vehicle is derived in sections III. The multi-hop uplink and downlink connectivity probabilities for infrastructure-based VANETs are derived in section IV. Analytical, simulation and experiment results are presented in section V, followed by conclusions in section VI.

\section{System ModeL}

The abstracted network scenario is presented in Fig. 1. In this figure, roadside infrastructure (Base stations) is built along the road, such as $\mathrm{BS}_{1}$ and $\mathrm{BS}_{2} . A, B, C, \ldots$, and $H$ are vehicles traveling on the road segment ${ }^{1}$. Here let $L$ be length of the road segment in meters.

Multi-hop relay is allowed in this network, and thus vehicles without direct link to BSs will have connections to BSs if they can find multi-hop relays (e.g., $D$ can access $\mathrm{BS}_{1}$ via $C, B$, and $A^{2}$, and be accessed by $\mathrm{BS}_{1}$ via $B$ and $C$ ). Vehicles that have no multi-hop connections to BSs, such as $E$ are isolated vehicles. We assume there are some existing protocols to allow vehicle to choose one of their neighboring vehicles with minimum-hop connections to BSs be the next hop relay (e.g., $F$ will choose $H$ instead of $G$ as its next hop relay, and it can access $\mathrm{BS}_{2}$ via just two hops), and doppler shift has been compensated with some schemes, e.g. [14]. To guarantee the delay performance in VANETs, a maximum number of hops a packet can travel is assumed to be $K$.

For traffic flow models, first, Poisson distribution is used to determine the random number of vehicles in our analysis, where the probability that there are $n$ vehicles on the road segment is

$$
f(n, L)=\frac{(\rho L)^{n} e^{-\rho L}}{n !}
$$

where $\rho$ is the traffic density, defined as cars per meter.

This distribution has been partly verified by our field test. During the field test, we collected real traffic data of S20 expressway in Shanghai from 0:00, Dec. 19 to 24:00, Dec. 20,2010 , CST. The number and average speed of vehicles crossing the testing point were recorded every twenty seconds. A histogram of number of cars (during a two-hour interval in the evening) is shown in Fig. 2. From this figure, it can be seen that the distribution of the number of vehicles approximately follows Poisson distribution. Finally, given the number of vehicles on the road, the vehicles are uniformly distributed along the road segment.

\footnotetext{
${ }^{1}$ Road segment is a part of a road between two adjacent BSs.

${ }^{2}$ There is only an unidirectional connection from $\mathrm{BS}_{1}$ to $B$, as $\mathrm{BS}_{1}$ is not in the coverage radius of $B$.
} 


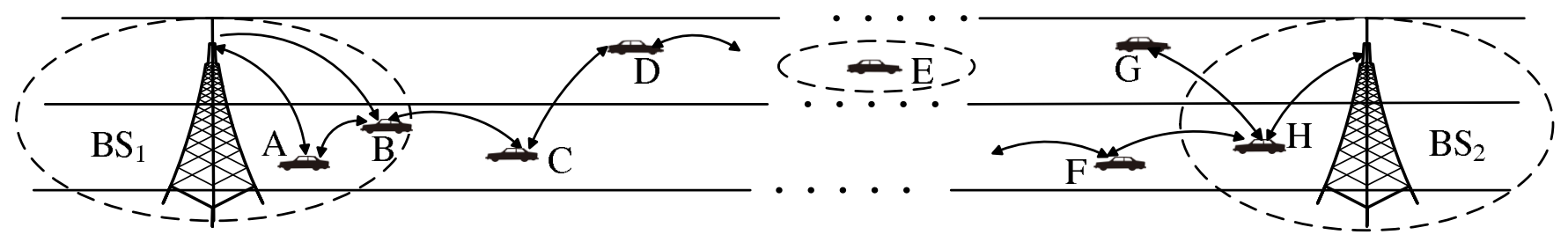

Fig. 1. Scenario of VANET along the road segment.

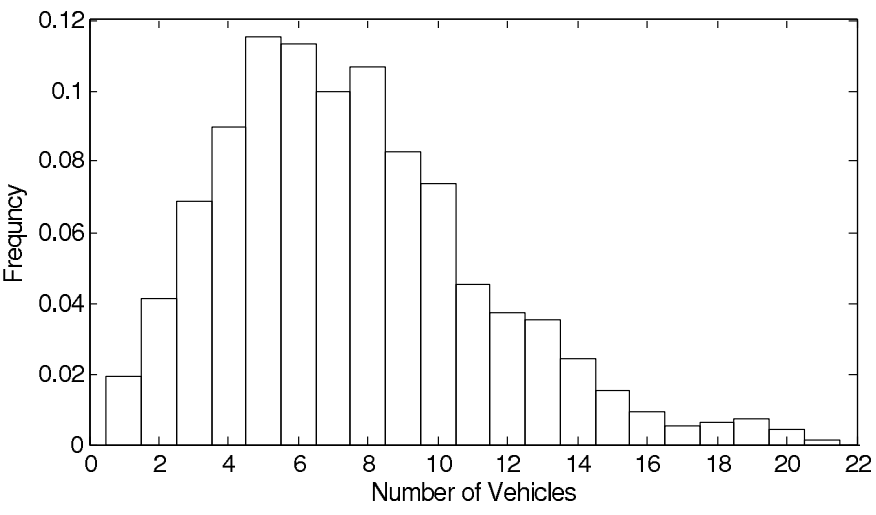

Fig. 2. Histogram of S20 Traffic Flow.

For communication models, both the unit disk (protocol model) and log-normal shadow communication model ${ }^{3}$ are considered in this paper. For the unit disk model, any vehicle pair or vehicle-BS pair is able to be connected if the distance between each other is less than the coverage radius [17]. Expression for log-normal shadow model will be given in the following section. In our analysis, the capacity of communication modules at different vehicles are assumed to be identical and communication modules at different BSs stations are also assumed to be identical.

\section{Connectivity Probability Between an ARbitraRy PAIR OF BS AND Vehicle}

Define a $k$-hop neighbor of a BS to be a vehicle that is able to receive messages from the BS via exactly $k$ hops. Let $\Phi_{k}^{b}(x)$ be the probability of the event that "a vehicle $x$ meters away from a BS is a $k$-hop neighbor of the BS". Similarly, define a $k$-hop neighbor of a vehicle to be a BS (or a vehicle) that is able to receive messages from the vehicle via exactly $k$ hops. Let $\Phi_{k}^{v}(x)$ be the probability of the event that "a BS (or a vehicle) $x$ meters away from a specific vehicle is a $k$-hop neighbor of the vehicle". These two types of definitions are corresponding to downlink transmission (i.e., safety or entertainment information from BSs) and uplink transmission (i.e., feedback or request information from vehicles), respectively. It is worth noting that the communication capabilities of BSs and vehicles are usually different, so $\Phi_{k}^{b}(x)$ and $\Phi_{k}^{v}(x)$ are expected to be different. The formula for $\Phi_{k}^{b}(x)$ will be derived at first.

If a vehicle is a $k$-hop neighbor of a $\mathrm{BS}$, then $k$ should

${ }^{3}$ Large-scale fading is implicitly incorporated in this model. satisfy the following equation

$$
k=\left\{\begin{array}{l}
0, \text { if the vehicle has no connection to the } \mathrm{BS} \\
1, \text { if the vehicle has direct connection to the BS } \\
\min \{\text { hops of its neighbors }+1, \text { otherwise }
\end{array}\right.
$$

Lemma 1: A vehicle is a $k$-hop neighbor of a specific BS, if it is not $n$-hop $(0 \leq n<k)$ neighbor of the BS, and at least one of its neighbors is a $(k-1)$-hop neighbor of the BS.

Lemma 1 is obvious, and the proof is omitted.

$\Phi_{0}^{b}(x)$ is the probability that a vehicle $x$ meters away from a BS is isolated from the BS. Meaning of $\Phi_{1}^{b}(x)$ is clear, i.e., under protocol model, if a vehicle is in the coverage radius of a $\mathrm{BS}$, it is able to receive messages from the BS with $100 \%$, and thus it is $100 \%$ a 1-hop neighbor of the BS. If coverage radius of BSs is $R$, then $\Phi_{1}^{b}(x)$ can be expressed mathematically as follows,

$$
\Phi_{1}^{b}(x)= \begin{cases}1, & x<R \\ 0, & \text { otherwise }\end{cases}
$$

Under log-normal shadow model (physical model) which is more close to reality, from the following expression,

$$
p_{r}=p_{0}-10 \alpha \log _{10} \frac{x}{d_{0}}+N_{\sigma}
$$

where $p_{r}$ is the received power (in $\mathrm{dBmW}$ ) at target vehicle, $p_{0}$ is the power (in $\mathrm{dBmW}$ ) at a reference distance $d_{0}, \alpha$ is the path loss exponent, $N_{\sigma}$ is a Gaussian random variable with zero mean and variance $\sigma^{2}, x$ is the Euclidean distance between the BS and a vehicle, if threshold $\left(p_{t h}\right)$ of the received power for decoding at BSs is given, we have

$$
\Phi_{1}^{b}(x)=\operatorname{Pr}\left(p_{r}>p_{t h}\right)
$$

Now, we move to the stage of finding the value of $\Phi_{i}^{b}(x)$ where $i>1$ and present a lemma at first.

Lemma 2: The sum of $\Phi_{i}^{b}(x)(i=0,1,2, \ldots,+\infty)$ is 1 , i.e.,

$$
\sum_{i=0}^{+\infty} \Phi_{i}^{b}(x)=1
$$

Proof: For any vehicle $x$ meters away from a BS, denote $E_{i}(x)$ be the event that "a vehicle $x$ meters away from a BS is a $i$-hop neighbor of the BS". $E_{0}(x), E_{1}(x), E_{2}(x), \ldots$, and $E_{+\infty}(x)$ are mutually disjoint events, and all of them forms a sample space $\Omega$. Therefore,

$$
\operatorname{Pr}\left(\bigcup_{i=0}^{+\infty} E_{i}(x)\right)=\sum_{i=0}^{+\infty} \operatorname{Pr}\left(E_{i}(x)\right)=\sum_{i=0}^{+\infty} \Phi_{i}(x)=1
$$

After going through Lemma 1 and 2, we have the following proposition. 


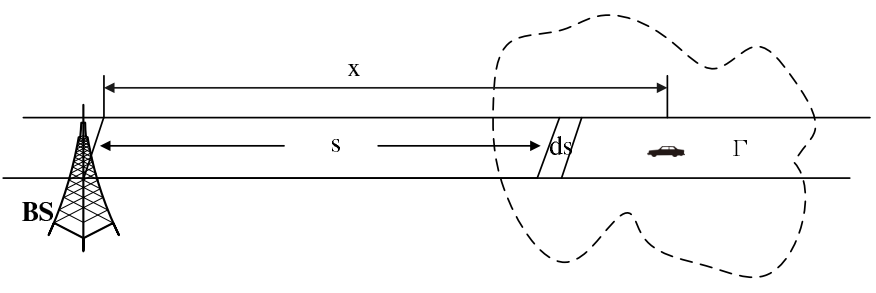

Fig. 3. Target vehicle $x$ meters away from a BS is $k$-hop neighbor of the BS.

Proposition 1: when $k>1$,

$$
\begin{aligned}
& \Phi_{k}^{b}(x)=\left(1-\sum_{i=0}^{k-1} \Phi_{i}^{b}(x)\right) \\
& \left(1-\exp \left(-\int_{\Gamma} \Phi_{k-1}^{b}(s) \cdot \Phi_{1}^{v}(x-s) \cdot \rho d s\right)\right)
\end{aligned}
$$

where $\Gamma$ is integration area, and $d s$ is integration increment ${ }^{4}$.

Proof: As shown in Fig. 3, area enclosed by dashed line and borders of road segment (denoted as $\Gamma$ ) is where possible neighbors of the target vehicle (a vehicle arbitrarily chosen for the analysis) could be found.

Set position of the BS as reference point. Then we start our analysis from a point that is $s$ meters away from the BS.

Lemma 1 tells us that a vehicle has to meet two requirements in order to define itself as $k$-hop neighbor of a specific BS. In fact, left part of the righthand side of (6) is expressed in terms of the first condition, i.e., the probability that the vehicle is not $0,1,2 \ldots$ and $(k-1)$-hop neighbor of the BS. Let $G(x)$ be the event that "for a specific vehicle $x$ meters away from the $\mathrm{BS}$, at least one of its neighbors is a $(k-1)$-hop neighbor of the BS". Then the right part of the righthand side of (6) is just the probability of $G(x)$, but it needs a bit explanation.

Let $g(s)$ be probability that no vehicle in $s \cap \Gamma$ is a $(k-1)$ hop neighbor of the BS. Then, $g(s+d s)$ is probability of the event that "no vehicle in $(s+d s) \cap \Gamma$ is a $(k-1)$-hop neighbor of the BS". This event is equivalent to the event that "there are no vehicles in $s \cap \Gamma$ and $d s$ is a $(k-1)$-hop neighbor of the BS". Let $f(d s)$ denote the probability that there is at least one vehicle traveling in $d s$, then we can express $g(s+d s)$ as

$$
g(s+d s)=g(s) \cdot\left(1-f(d s) \cdot \Phi_{k-1}^{b}(s) \cdot \Phi_{1}^{v}(x-s)\right)
$$

Following Poisson distribution, when $d s$ is infinitely small, we have

$$
f(d s)=\rho d s
$$

Then, re-arrange (7), we have a differential equation,

$$
\begin{aligned}
& \frac{g(s+d s)-g(s)}{d s}=-g(s) \cdot \Phi_{k-1}^{b}(s) \cdot \rho \cdot \Phi_{1}^{v}(x-s) \\
& \Rightarrow g^{\prime}(s)=-g(s) \cdot \Phi_{k-1}^{b}(s) \cdot \rho \cdot \Phi_{1}^{v}(x-s)
\end{aligned}
$$

Solve (9) and we have the probability of $\overline{G(x)}$

\footnotetext{
${ }^{4}$ In our analysis, for simplicity $\Gamma$ could be regarded as 1-dimensional integration interval, while actually, if distributions for traffic flows on different lanes are known, then we could modify (6) a little bit into double integration to support 2-dimensional scenarios.
}

$$
\begin{aligned}
\operatorname{Pr}(\overline{G(x)}) & =\int_{\Gamma} d g(s) d s \\
& =C \cdot \exp \left(-\int_{\Gamma} \Phi_{k-1}^{b}(s) \Phi_{1}^{v}(x-s) \rho d s\right)(10)
\end{aligned}
$$

Note the constant number $C$ equals 1 , as $\operatorname{Pr}(\overline{G(x)})$ should be in the interval $[0,1]$.

Effortlessly, we have the probability of $G(x)$

$$
\operatorname{Pr}(G(x))=1-\exp \left(-\int_{\Gamma} \Phi_{k-1}^{b}(s) \cdot \Phi_{1}^{v}(x-s) \cdot \rho d s\right)
$$

which is just the right part of righthand side of (6).

Similarly, the probability that a BS is a $k$-hop neighbor of a vehicle can be written as

$$
\begin{aligned}
& \Phi_{k}^{v}(x)=\left(1-\sum_{i=0}^{k-1} \Phi_{i}^{v}(x)\right) \\
& \left(1-\exp \left(-\int_{\Gamma} \Phi_{k-1}^{v}(s) \cdot \Phi_{1}^{v}(x-s) \rho d s\right)\right)
\end{aligned}
$$

where for unit disk model

$$
\Phi_{1}^{v}(x)= \begin{cases}1, & x<r \\ 0, & \text { otherwise }\end{cases}
$$

, and for log-normal model, with (3), we have

$$
\Phi_{1}^{v}(x)=\operatorname{Pr}\left(p_{r}>p_{t h}^{\prime}\right)
$$

where $p_{t h}^{\prime}$ is threshold of received power for decoding at the vehicles.

For simplicity, $\Phi_{0}^{b}(x)$ is approximated to be probability that the BS has no 1-hop neighbors, and $\Phi_{0}^{v}(x)$ is approximated to be probability that the target vehicle has no 1-hop neighbors. Note that since $\Phi_{1}^{b}(x)$ and $\Phi_{1}^{v}(x)$ are different, values for (6) and (12) are expected to be different. For some other traffic and communication models, if $f(d s), \Phi_{1}^{v}(x)$ and $\Phi_{1}^{b}(x)$ are known, then (6) and (12) can be easily extended. And for $\Phi_{k}^{v}(x)$, it can be regarded as the connectivity probability formula for pure ad-hoc-like networks.

\section{Connectivity Probability for Multi-Hop INFRASTRUCTURE-BASED VANETS}

Rationale behind the difference in the definitions for uplink and downlink connectivity probability is that capacities (transmit/receive radii) of BSs and vehicles are different and safety information broadcasting in VANETs has the highest priority, so for downlink transmission, how to guarantee that all vehicles are able to receive safety information should be focused, while for uplink transmission, it is not that strict ${ }^{5}$ (e.g., if $98 \%$ of messages from vehicles can be received by the infrastructure, then uplink connectivity performance may also be regarded as good).

\footnotetext{
${ }^{5}$ For pure VANETs, since every node (vehicle) in the network is identical, there are no uplink and downlink differences.
} 


\section{A. Uplink Connectivity Probability}

The uplink connectivity probability is presented in the following proposition.

Proposition 2: Multi-hop uplink connectivity probability for infrastructure based VANETs is

$$
p_{u}=\int_{0}^{L} \frac{d x}{L}\left\{1-\left(1-\sum_{i=1}^{K} \Phi_{i}^{v}(x)\right)\left(1-\sum_{i=1}^{K} \Phi_{i}^{v}(L-x)\right)\right\}
$$

Proof: Let $H(x)$ be the event that "messages from a vehicle $x$ meters from $\mathrm{BS}_{1}$ can be received by the infrastructure". If $H(x)$ is true, then "either $\mathrm{BS}_{1}$ or $\mathrm{BS}_{2}$ or they both are $n$-hop $(n=1,2, \ldots$, or $K)$ neighbors of the vehicle". Thus the probability of $H(x)$ is

$$
\operatorname{Pr}(H(x))=1-\left(1-\sum_{i=1}^{K} \Phi_{i}^{v}(x)\right)\left(1-\sum_{i=1}^{K} \Phi_{i}^{v}(L-x)\right)
$$

The uplink connectivity probability can be regarded as the expectation of the probability that "messages from an arbitrary vehicle can be received by the infrastructure". Hence the uplink connectivity probability is

$$
p_{u}=\int_{0}^{L} \operatorname{Pr}(H(x)) \frac{d x}{L}
$$

and thus (15) is obtained.

\section{B. Downlink Connectivity Probability}

We divided the road segment equally into two parts: left and right part. If messages can be broadcast from infrastructure to all vehicles on the road segment, then approximately all vehicles traveling on the left part can be accessed by $\mathrm{BS}_{1}$ (i.e., messages from $\mathrm{BS}_{1}$ can reach these vehicles) and all vehicles on the right part can be accessed by $\mathrm{BS}_{2}$.

Lemma 3: The probability that all vehicles traveling on the right part can be accessed by $\mathrm{BS}_{2}$ is approximately the probability that vehicle farthest to $\mathrm{BS}_{2}$ can be accessed by $\mathrm{BS}_{2}$.

Proof: $\Phi_{k}^{v}(x)$ contains information about connection conditions with neighboring vehicles, and thus vehicles traveling on the right part farthest to $\mathrm{BS}_{2}$ has the least probability to be accessed by $\mathrm{BS}_{2}$. If that vehicle can be accessed by $\mathrm{BS}_{2}$, then we can assume all vehicles on the right half can be accessed by $\mathrm{BS}_{2}$.

With Lemma 3, we have the following proposition,

Proposition 3: Multi-hop downlink connectivity probability for infrastructure based VANETs is

$$
p_{d}=\left\{e^{-\frac{\rho L}{2}}+\int_{0}^{\frac{L}{2}} \rho d u \cdot e^{-\rho u} \cdot\left(\sum_{i=1}^{K} \Phi_{i}^{b}\left(\frac{L}{2}-u\right)\right)\right\}^{2}
$$

Proof: We derive the downlink connectivity probability for the right part at first. As shown in Fig. 4, the central line of the road segment is set as reference, and direction $\mathrm{BS}_{1} \rightarrow$ $\mathrm{BS}_{2}$ is set as positive direction.

Define $T(u)$ as the event that "the farthest vehicle which can be accessed by $\mathrm{BS}_{2}$ in the right part lies in $(u, u+d u)$ ". More intuitively, $T(u)$ is equivalent to the event that "there

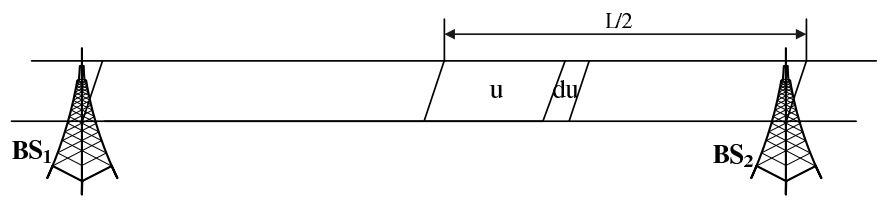

Fig. 4. The farthest vehicle to $\mathrm{BS}_{2}$ lies in the area $(u, u+d u)$.

are no vehicles in $(0, u)$, and there is one $n$-hop $(n=1,2, \ldots$, or $K)$ neighbor of $\mathrm{BS}_{2}$ in $(u, u+d u)$ ". With (1) we have

$$
\operatorname{Pr}(T(u))=e^{-\rho u} \cdot \rho d u \cdot\left(\sum_{i=1}^{K} \Phi_{i}^{b}\left(\frac{L}{2}-u\right)\right)
$$

If there are no vehicles traveling on the right part of the road segment, network along the right part is also be considered as fully connected. Denoted this event as $\kappa$, according to (1), the probability of $\kappa$ is

$$
\operatorname{Pr}(\kappa)=e^{-\frac{\rho L}{2}}
$$

Derivation for the left half is the same, and they are independent to each other. Thus finally we will have (18).

\section{Numerical Results AND Discussions}

Both computer-based simulations and experiments (realtraffic-flow-based simulations) have been done to validate our analytical results. For the computer-based simulations, 10000 Poisson distribution realizations were generated to indicate positions of vehicles on the road segment. Then the downlink and uplink connectivity probabilities were calculated during each realization and averaged over all realizations. For the experiments, four hidden radars were installed on expressway S20 in Shanghai to monitor traffic flows. The number and average speed of vehicles crossing the testing point were recorded every twenty seconds for eight days. Average traffic density $\rho$ were calculated to be 0.0350 and 0.0845 cars per meters during non-busy periods (from 22:00 to 23:59, Dec. 19, 2010, CST) and busy periods (from 12:32 to 16:42, Dec. 19, 2010 CST), respectively. Analytical results were derived from equations (15) and (18). Different vehicle traffic densities, inter-BS distances, radio coverage ranges (BS and vehicle), and multi-hop communication capabilities were considered.

Fig. 5 (a) and (b) show the uplink connectivity probabilities $p_{u}$ during non-busy and busy periods as a function of interBS distance $L$ under unit disk model. In both cases, simulation and experiment results match very well with analytical results under different multi-hop communications conditions. Obviously, single-hop uplink connectivity probability achieves $100 \%$ when $L$ is less than $2 r$ (1000 meters). In multi-hop communications, the probability of finding multiple relays (one for each hop) declines when the number of hops increases. So the turning points for $K$-hop $(K=2,3,4)$ uplink connectivity probability curves are slightly less than $2 K r$ in both (a) and (b) ${ }^{6}$. When inter-BS distance $L$ becomes larger than these turning points, the corresponding $p_{u}$ curves are monotonically

\footnotetext{
${ }^{6}$ During busy periods, number of neighboring vehicles is larger, thus the probability of finding multi-hop relays is higher, so turning points in (b) are larger than that in (a).
} 


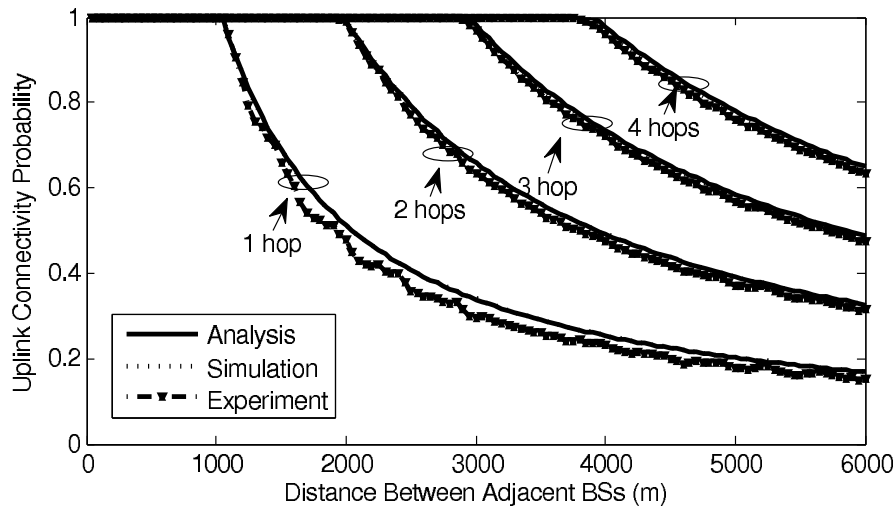

(a) Non-busy Periods

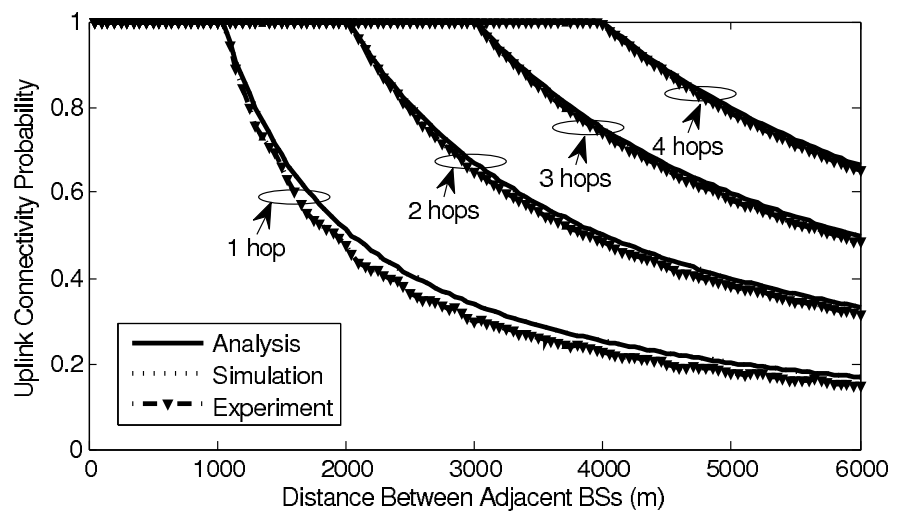

(b) Busy Periods

Fig. 5. Uplink Connectivity Probability, $R=1000 \mathrm{~m}, r=500 \mathrm{~m}$ (under unit disk model).

decreasing in parallel, with a vertical gap of $2 r / L$ between adjacent curves. So network designers can effectively control and manage uplink connectivity probability performance for vehicles/end-users by applying gradual impact from inter-BS distance $L$ (related to network investment) and/or stepwise impact from hop number $K$ (related to operation complexity).

Fig. 6 (a) and (b) show the downlink connectivity probabilities $p_{d}$ as function of inter-BS distance $L$ under unit disk model. As seen, analytical, simulation and experiment results are better matched in (b) than that in (a). Turning points of experiment curve for 4-hop $p_{d}$ in (a) deviates that of analytical and simulation curves a little bit. Similar as turning points in Fig. 5, the falling-down edges of for single-hop and multi-hop analytical and simulation curves are located at $L=2 R+2(K-1) r$ (slightly to the left due to the same reasons as in Fig. 5). These sharp edges indicate the technical challenges, key system parameters and basic rules in designing and realizing a fully connected vehicular network.

Form $p_{u}$ and $p_{d}$ curves in Figs. 5 and 6, it is seen that the turning points for the downlink connectivity curves start from $2 R(2000 \mathrm{~m})$ while turning points for uplink connectivity start from $2 r(1000 \mathrm{~m})$, which indicates that if $R>r$, then downlink transmission is more reliable than uplink transmission in the same network. Also $p_{u}$ and $p_{d}$ curves in Figs. 5 and 6 demonstrate the accuracy of our analytical approach and results, i.e., (15) and (18), which can effectively guide the real-world practice of vehicular network design, implementation and management. In particular, they enable network designers and operators to achieve and guarantee predefined performance targets, in terms of the uplink and the downlink connectivity probabilities, under different vehicle traffic density, radio coverage and multi-hop communications conditions. The horizontal gaps $2 r$ between adjacent turning points (Fig. 5) and adjacent edges (Fig. 6) reveal the trade-off between hop number $K$ and inter-BS distance $L$ under the same connectivity performance. Specifically, without affecting the $100 \%$ connectivity probability performance, $L$ can be extended by a distance of $2 r$ (meaning less BSs and less investment) when one more hop is allowed in vehicular communication networks ${ }^{7}$.

\footnotetext{
${ }^{7}$ This conclusion is valid when traffic density $\rho$ is sufficiently large.
}

Fig. 7 (a) and (b) shows the uplink connectivity probability performance as a function of radio coverage range $r$ of a vehicle under unit disk model. When $r$ is less than $1 / \rho$, it is hard to find any neighboring vehicles as a relay, so the uplink connectivity probability curves for different multi-hop capabilities are indistinguishable in the range of $[0,1 / \rho]$. As $r$ increases, the advantages of multi-hop communications get clearer. The exact locations of the saturation points for these curves reaching the maximum $100 \%$ uplink connectivity probability can be estimated as $r_{s}=L /(2 K)+(K-1) /(K \rho), K=1$, $2,3,4$.

Fig. 8 (a) and (b) shows the downlink connectivity probability as a function of vehicle coverage range $r$ under unit disk model. The sharp rising edges of these curves indicate the critical conditions for realizing a fully connected vehicular network. Note that the rising edge of single-hop curve is located at $r=1250$ meters and, therefore, not presented in Fig. 8. These two figures clearly demonstrate the trade-off between service performance, in terms of downlink connectivity probability, and key system parameters, i.e., hop number $K$ and vehicle coverage range $r$, which determine the operation complexity and transmission power of vehicles. Comparing (a) and (b), it is seen that turning-points in (b) are smaller than that in (a), which indicates that when traffic density $\rho$ is high, without impacting the $100 \%$ downlink connectivity probability, vehicle coverage range $r$ can be decreased to save energy and reduce interference. Moreover, comparing (a) and (b), it is seen that the experiment results for non-busy periods have higher attenuations, which is due to that vehicle density is lower in non-busy periods, and as each vehicle has fewer neighboring vehicles (statistically), it is more difficult to identify relays for establishing a connection with a roadside BS.

The impact of inter-vehicle distance, i.e., the inverse of traffic density parameter $\rho$, on the uplink connectivity probability performance is shown in Fig. 9 for different multi-hop conditions under unit disk model. Considering a specific vehicle, single-hop uplink connectivity probability curve depends on its location and is therefore fixed at $2 r / L=0.4$. Multi-hop $p_{u}$ curves start from the values of $\min \{1,2 K r / L\}$ (when traffic density is very high) and are then monotonically decreasing as inter-vehicle distance increases, which means fewer and fewer 


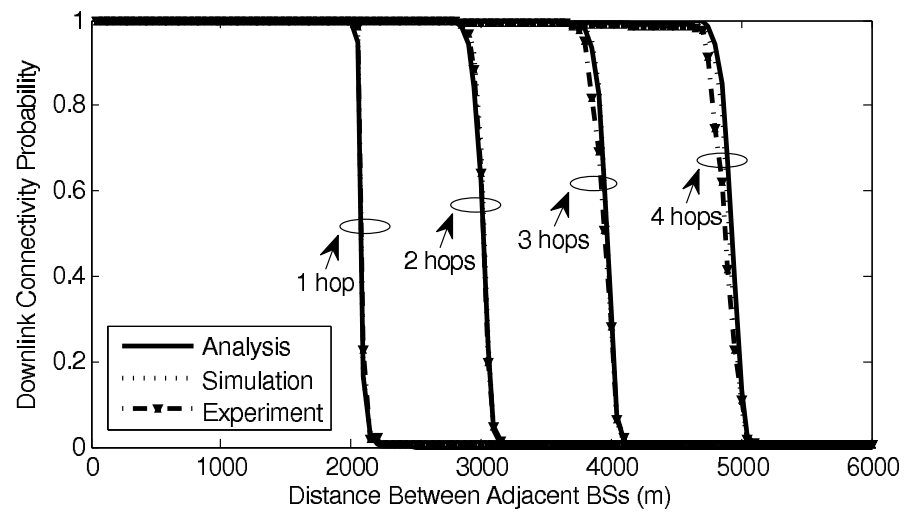

(a) Non-busy Periods

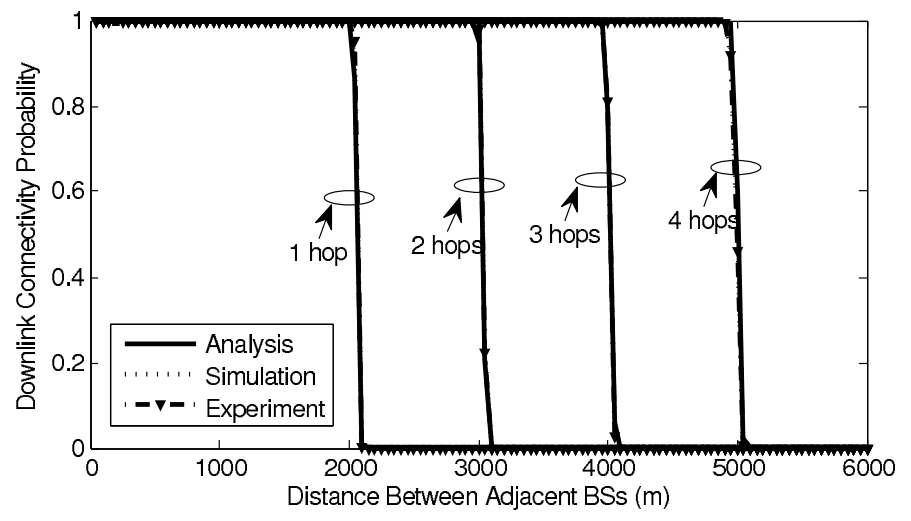

(b) Busy Periods

Fig. 6. Downlink Connectivity Probability, $R=1000 \mathrm{~m}, r=500 \mathrm{~m}$ (under unit disk model).

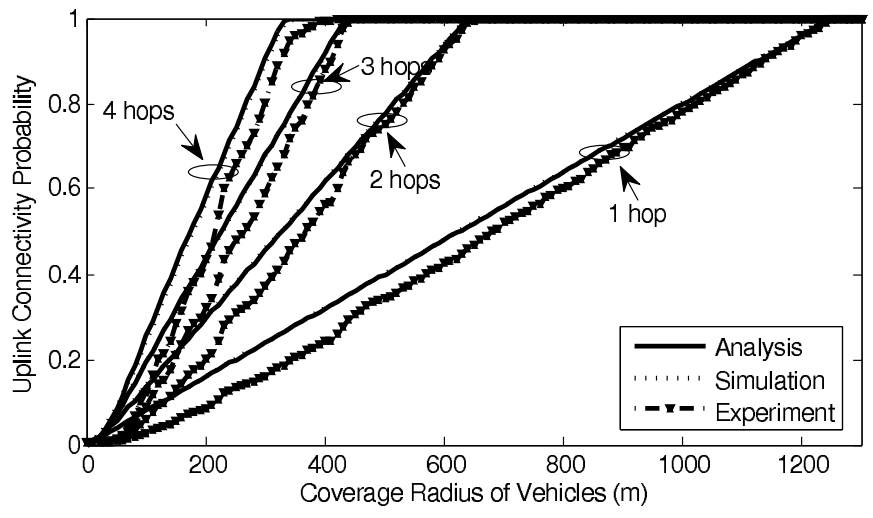

(a) Non-busy Periods

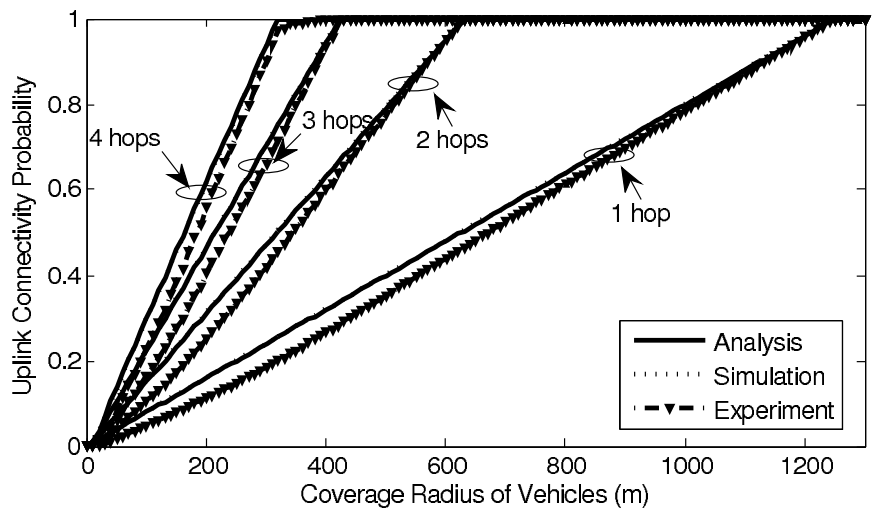

(b) Busy Periods

Fig. 7. Uplink Connectivity Probability, $L=2500 \mathrm{~m}, R=1000 \mathrm{~m}$ (under unit disk model).

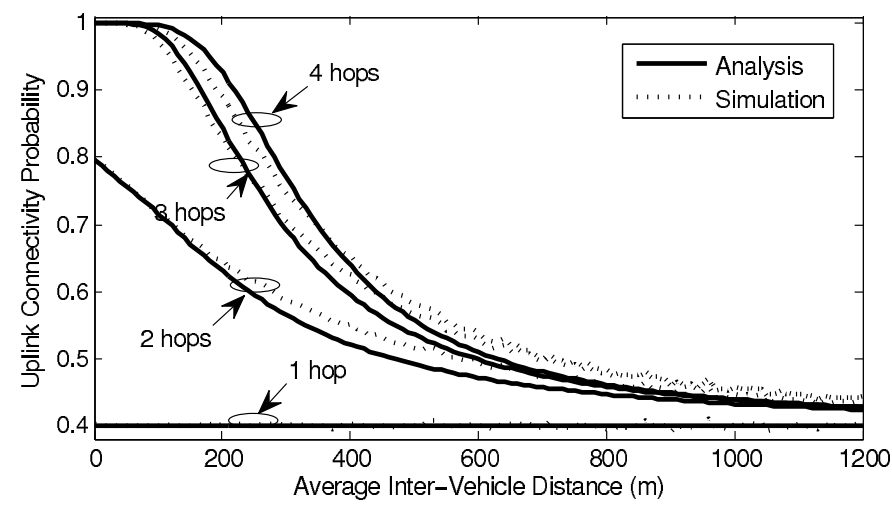

Fig. 9. Uplink Connectivity Probability, $L=2500 \mathrm{~m}, R=1200 \mathrm{~m}, r=500 \mathrm{~m}$ (under unit disk model).

neighboring vehicles can be found and used as relay.

By using Figs. 8-9 as a practical guidance, a network designer or operator can effectively conduct (through roadside BSs) dynamic transmission power control among neighboring vehicles, thus to minimize energy consumption, interference and multi-hop complexity while achieving the predefined network service performance.

Fig. 10 shows comparisons between analytical results of the downlink connectivity probability under unit disk model with that under log-normal shadow model during non-busy periods.

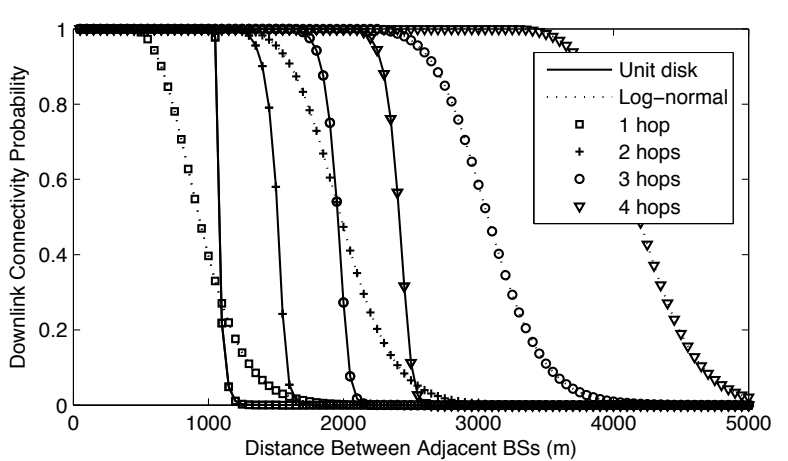

Fig. 10. Downlink Connectivity Probability, $L=2500 \mathrm{~m}, R=1000 \mathrm{~m}, r=$ $500 \mathrm{~m}$ (Comparisons under unit disk model and log-normal shadow model).

Here, $\alpha=3, \sigma=8 \mathrm{~dB}, \rho=0.015$ cars/meter. As seen, the downlink connectivity curves for unit disk model have sharper falling-down edges. The single-hop downlink connectivity curve for unit disk model indicates better performance when $L<2000$, while for the multi-hop downlink connectivity probability curves, results under log-normal shadow model outstrip that under unit disk model, which indicates that for multi-hop VANETs, shadow increases connectivity probability. Moreover, when $\sigma$ is zero, curves under both models are the same, and thus, results under unit disk model can be 


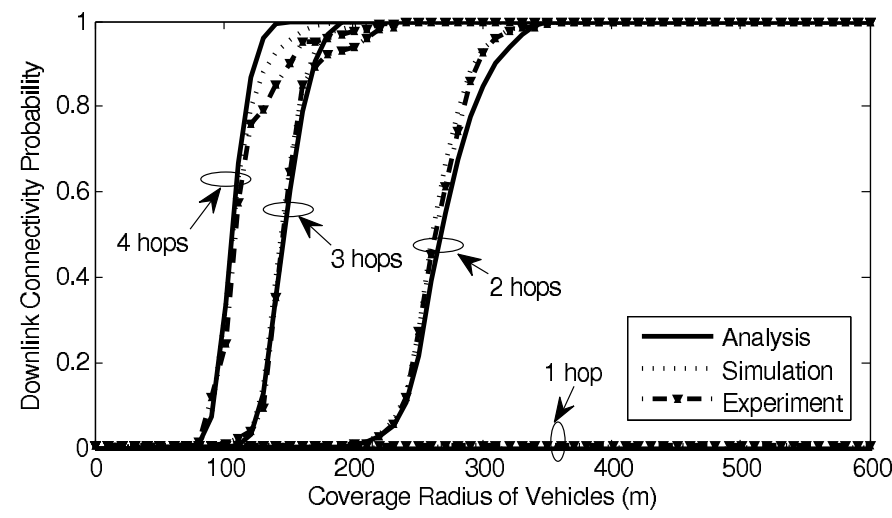

(a) Non-busy Periods

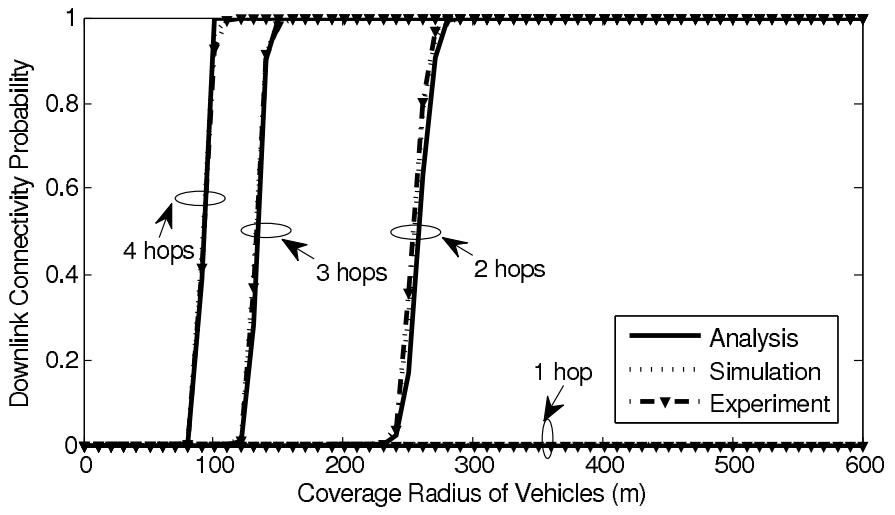

(b) Busy Periods

Fig. 8. Downlink Connectivity Probability, $L=2500 \mathrm{~m}, R=1000 \mathrm{~m}$ (under unit disk model).

regarded as special cases of that under log-normal shadow model.

\section{CONCLUSIONS}

In this paper, an analytical model supporting multi-hop relay for infrastructure-based vehicular networks was proposed. Based on this model, formulae for multi-hop uplink and downlink connectivity probabilities were derived. These analytical results, verified by computer simulations and experiments, reveal the trade-off between these key performance metrics and some important system parameters, such as BS and vehicle densities, radio coverage (or transmission power), and the maximum number of hops a path contains. This insight knowledge enables vehicular network engineers and operators to effectively achieve high user satisfaction and good service coverage, with only necessary deployment of BSs along the road according to traffic density, user requirements and service types. Our analytical approach and results will be extended by considering more practical traffic flow models.

\section{ACKNOWLEDGMENT}

This research is partially supported by the National Natural Science Foundation of China (NSFC) under the grant 60902041, the Ministry of Science and Technology (MOST) of China under grants 2009DFB13080, 2009ZX03003-009, and 2010ZX03005-003, the Research Fund of National Mobile Communications Research Laboratory, Southeast University (No. 2011A04), the Research Councils UK (RCUK) under the UK-China Science Bridges Project: R\&D on (B) 4 G Wireless Mobile Communications (EP/G042713/1), the European Commission under the FP7 project: Cooperating Objects Network of Excellence (CONET, FP7-2007-2-224053), and ARC Discovery projects: DP110100538 and DP120102030.

\section{REFERENCES}

[1] "Website of National Bureau of Statistics of China," (Access Date: May 14, 2011) http://www.stats.gov.cn/tjgb/.

[2] W. Niu, "Report of New Urbanization in China 2010," Science Press, June 2010.

[3] "IEEE P802.11- task group p," (Access Date: May 14, 2011) http://grouper.ieee.org/groups/802/11/Reports/tgp_update.htm.
[4] "IEEE 1609-family of standards for wireless access in vehicular environments(WAVE)," (Access Date: May 14, 2011) http://www.standards.its.dot.gov/fact_sheet.asp?f $=80$.

[5] Y. Zhou, Z.G. Pan, J.L. Hu, et al, "Broadband Wireless Communications on High Speed Trains," in Proc. IEEE Wireless and Optical Communications Conference (WOCC), pp. 1-6, Apr. 2011.

[6] A. Abdrabou, W. Zhuang, "Probabilistic Delay Control and Road Side Unit Placement for Vehicular Ad Hoc Networks with Disrupted Connectivity," IEEE J. Sel. Areas Commun., Vol. 29, No. 1, pp. 129139, Jan. 2011.

[7] C. Foh, and B. Lee, "A close form network connectivity formula for one-dimensional MANETs," in Proc. IEEE International Conference on Communications, Vol. 6, pp. 3739-3742, June 2004.

[8] S. Ukkusuria, and L. Dub, "Geometric connectivity of vehicular ad-hoc networks: Analytical characterization," Transportation Research Part C: Emerging Technologies, Vol. 16, No. 5, pp. 615-634, Oct. 2008.

[9] D. Miorandi, and E. Altman, "Connectivity in one-dimensional ad-hoc networks: a queueing theoretical approach," Wireless Networks, Vol. 12, No. 5, pp. 573-587, Sept. 2006.

[10] J. Wu, "Connectivity analysis of a mobile vehicular ad-hoc network with dynamic node population," in Proc. IEEE Global Telecommunications Conference, Dec. 2008.

[11] O. Dousse, P. Thiran, and M. Hasler, "Connectivity in ad-hoc and hybrid networks," in Proc. IEEE International Conference on Computer Communications, Vol.2, pp. 1079-1088, June 2002.

[12] S. Ng, and G. Mao, "Analysis of k-Hop Connectivity Probability in 2-D Wireless Networks with Infrastructure Support," in Proc. IEEE Global Telecommunications Conference, pp. 1-5, Jan. 2011.

[13] S. Ng, W. Zhang, Y. Zhang, Y. Yang, and G. Mao, "Analysis of Access and Connectivity Probabilities in Vehicular Relay Networks," IEEE J. Sel. Areas Commun., Vol. 29, No. 1, pp. 140-150, Jan. 2011.

[14] Y. Zhou, J. Wang, and M. Sawahashi, "Downlink transmission of broadband OFCDM systems - Part II: Effect of Doppler Shift", IEEE Trans. Commun., Vol. COM-54, No. 6, pp. 1097-1108, June 2006.

[15] X. Ta, G. Mao, and B. Anderson, "On the probability of k-hop connection wireless sensor networks," IEEE Commun. Lett., Vol. 11, No. 8, pp. 662-664, Aug. 2007.

[16] A. Kothari, and D. Patel, "Methodology to Solve the Count-To-Infinity Problem by Accepting and Forwarding Correct and Updated Information Only Using "Test" Packet," in Proc. IEEE Advance Computing Conference, pp. 26-31, Mar. 2009.

[17] P. Wan and C. Yi, "Coverage by randomly deployed wireless sensor networks," IEEE Trans. Inf. Theory, Vol. 52, No. 6, pp. 2658-2669, June 2006.

[18] T. Rappaport, "Wireless communications: principles and practice," Prentice Hall PTR, 2nd, 2002.

[19] P. Gupta, and P. Kumar, "The Capacity of Wireless Networks," IEEE Trans. Inf. Theory, Vol. 46, No. 2, pp. 388-404, Mar. 2000.

[20] T. Camp, J. Boleng, and V. Davies, "A Survey of Mobility Models for ad-hoc Network Research," IEEE Wireless Communications and Mobile Computing, Vol. 2, No. 5, pp. 483C502, Sept. 2002.

[21] M. Kafsi, P. Papadimitratos, O. Dousse et al., "VANET Connectivity Analysis," in proceedings of Auto motive Networking and Applications Workshop, 2008. 
[22] R. Roy, "Handbook of Mobile ad-hoc Networks for Mobility Models," Part 1, Hardcover, pp. 23-32, 2011

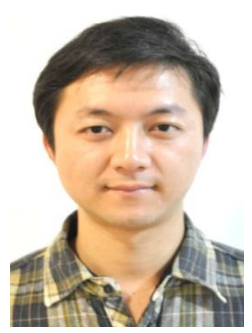

Wuxiong Zhang received the BEng degree in Information Security from Shanghai Jiao Tong University (SJTU), Shanghai, China, in 2008. He is now a $\mathrm{PhD}$ candidate of communication and information system in Shanghai Research Center for Wireless Communications, SIMIT, the Chinese Academic of Sciences. His research interests include beyond 3G mobile communication systems and vehicular networks.

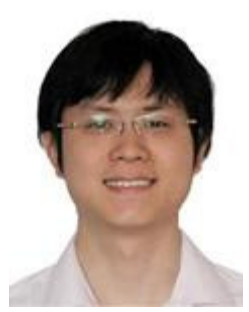

Yu Chen received the BEng degree in Electronic Science and Technology from Beijing University of Posts and Telecommunications (BUPT), Beijing, China, in 2006 and the MSc degree in Telecommunications from University College London (UCL), London, UK, in 2007, where he is now working toward the $\mathrm{PhD}$ degree. His general research interests include queueing theory, statistics and beyond $3 \mathrm{G}$ mobile communication systems.

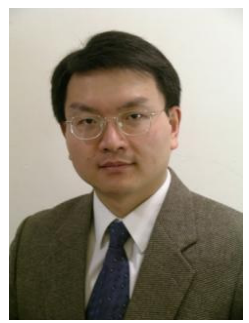

Yang Yang received the BEng and MEng degrees in Radio Engineering from Southeast University, Nanjing, P. R. China, in 1996 and 1999, respectively; and the $\mathrm{PhD}$ degree in Information Engineering from The Chinese University of Hong Kong in 2002. He is currently a professor at Shanghai Institute of Microsystem and Information Technology (SIMIT), Chinese Academy of Sciences, and serving as the Vice Director at Shanghai Research Center for Wireless Communications (WiCO). Prior to that, he served the Department of Electronic and Electrical Engineering at University College London (UCL), United Kingdom, as a Senior Lecturer, the Department of Electronic and Computer Engineering at Brunel University, United Kingdom, as a Lecturer, and the Department of Information Engineering at The Chinese University of Hong Kong as an Assistant Professor. His general research interests include wireless ad hoc and sensor networks, wireless mesh networks, $4 \mathrm{G}$ mobile communication systems, green and cooperative communications.

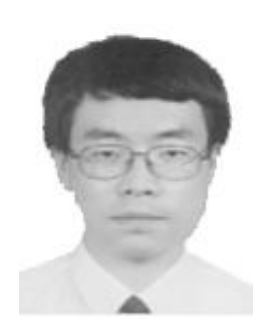

Xiangyang Wang received the $\mathrm{Ph} . \mathrm{D}$. degree in electrical engineering from the University of Hong Kong, Hong Kong, in 2003. He is with the National Mobile Communications Research Laboratory, Southeast University. His research interests include mobile communication systems, space-time signal processing, B3G/4G system, and the VLSI implementation of wireless systems.

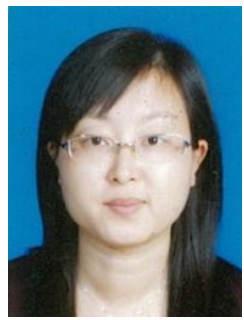

Yu Zhang received her Ph.D. in Computing from Imperial College London, U.K. in 2009. Currently she is a research staff with the National Engineering Research Center for Broadband Networks and Applications in Shanghai, China. Her main research areas of interest are related to performance analysis, modeling and simulation of telecommunication systems, vehicular ad hoc networks, and digital interactive TV.

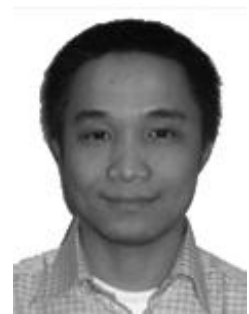

Xuemin Hong received his BSc degree in Communication Engineering from Beijing Information Science and Technology University, China, in 2004 and his Ph.D. degree in Wireless Communications from Heriot-Watt University, UK, in 2008. Since July 2011, he has been an Associate Professor with the School of Information Science and Technology, Xiamen University, China. His research interests include MIMO and cooperative systems, ultra wide band (UWB) systems, wireless radio channel modeling, cognitive radio networks, and (beyond) $4 \mathrm{G}$ wireless communications.

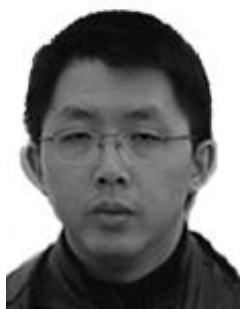

Guoqiang Mao received the Ph.D. degree in telecommunications engineering in 2002 from Edith Cowan University. He joined the School of Electrical and Information Engineering, the University of Sydney in December 2002 where he is a Senior Lecturer now. His research interests include wireless localization techniques, wireless multihop networks, graph theory and its application in networking, and network performance analysis. 